\title{
Calcaneal Tuberosity
}

National Cancer Institute

\section{Source}

National Cancer Institute. Calcaneal Tuberosity. NCI Thesaurus. Code C142295.

A roughened surface on the superior portion of the posterior half of the calcaneus, where the calcaneal (Achilles) tendon inserts. 